Disponível em

http://www.anpad.org.br/rac

RAC, Curitiba, v. 15, n. 5, art. 7, pp. $918-930$, Set./Out. 2011

$((c)$ EY-NG

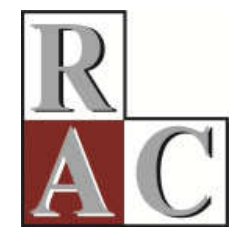

\title{
É Possível Bater o Ibovespa com Operações de Análise Técnica no Mercado Futuro?
}

\author{
Is It Possible to Outperform Ibovespa through Technical Analysis in the \\ Futures Market?
}

Diego Paraiso Garcia Guimarães * E-mail: diego@tfgr.com.br Associação Nacional das Instituições do Mercado Financeiro - ANDIMA Rio de Janeiro, RJ, Brasil.

Gustavo Silva Araújo

E-mail: gustavo.araujo@bcb.gov.br Instituto Brasileiro de Mercado de Capitais - IBMEC-RJ Rio de Janeiro, RJ, Brasil.

\section{Claudio Henrique da Silveira Barbedo} E-mail: cbarbedo@ibmecrj.br Instituto Brasileiro de Mercado de Capitais - IBMEC-RJ Rio de Janeiro, RJ, Brasil.

* Endereço: Diego Paraiso Garcia Guimarães

Av. das Américas, 3500, Ed. Hong Kong 1000, sala 415, Barra da Tijuca, Rio de Janeiro/RJ, 22640-102.

Copyright (C) 2011 RAC. Todos os direitos, até mesmo de tradução, são reservados. É permitido citar parte de artigos sem autorização prévia, desde que seja identificada a fonte. 


\title{
Resumo
}

O processo de seleção de ativos tem sido um dos principais desafios enfrentados por analistas do mercado financeiro. A partir da teoria formulada por Charles Dow, em 1884, diversos estudos se preocuparam com a questão da previsão de preços. Entretanto a teoria de eficiência de mercado desenvolve a ideia de que essa previsão é improfícua. O objetivo deste trabalho é verificar se padrões das séries históricas são capazes de proporcionar bons resultados para a realização de ganhos com o minicontrato de Ibovespa futuro. Utilizando os anos de 2006 e 2007 para calibragem da estratégia, e o período de janeiro de 2008 a fevereiro de 2010 para a sua aplicação, obtemos resultados muito superiores ao Ibovespa, mesmo quando o retorno é controlado pelo risco. Estes resultados sugerem que a forma fraca de eficiência de mercado é contrariada para o ativo no período estudado.

Palavras-chave: análise técnica; investimento; IBOVESPA.

\begin{abstract}
The asset selection decision has been one of the main challenges faced by financial market analysts. The theory of Charles Dow formulated in 1884 attempts to shed some light on this issue by forecasting stock market prices based on patterns. However the efficient market hypothesis has developed the hypothesis that this idea as useless. The aim of this work is to verify whether it is possible to profit from patterns in stock prices using the Ibovespa futures mini-contracts. Using the years 2006 and 2007 to calibrate the strategy and the period ranging from January 2008 to February 2010 to apply it, we obtained results higher than those of Ibovespa, even in terms of risk-return. The results suggest the weak form efficiency is not confirmed for the period under analysis.
\end{abstract}

Key words: technical analysis; investment; IBOVESPA. 


\section{Introdução}

O processo de seleção de ativos tem sido um dos principais desafios enfrentados por participantes do mercado financeiro. A partir da teoria formulada por Charles Dow, em 1884, diversos estudos se preocuparam com a questão da previsão de preços. Os métodos mais tradicionais de previsão utilizam ferramentas estatísticas, intuição ou julgamento de analistas. Working (1934), Cowles (1933), Cowles e Jones (1937) e Kendall (1953) demonstram a impossibilidade de se determinar a direção dos preços. Nestes trabalhos, os autores constatam que os preços das ações norteamericanas flutuam aleatoriamente, de modo a não colaborar para a previsão e a realização de ganhos.

Samuelson (1965) e Mandelbrot (1966) interpretaram estes resultados como prova de que os mercados financeiros funcionavam bem. Com isso a teoria de eficiência do mercado, sistematizada inicialmente por Roberts (1967) e aperfeiçoada por Fama (1970, 1991), surge com base na ideia de que as informações relevantes são incorporadas de forma imediata e correta aos preços dos ativos financeiros. Por esta teoria, a análise técnica ou o estudo do movimento do mercado, por meio de gráficos com o propósito de prever as tendências futuras dos preços, não pode ser bem sucedido, pelo fato de contrariar a eficiência fraca de Fama (1970).

O objetivo deste trabalho é verificar se padrões das séries históricas são capazes de proporcionar bons resultados para a realização de ganhos com o minicontrato de Índice Bovespa futuro ${ }^{(1)}$. Segundo a literatura da área, se bons resultados podem ser obtidos, a forma fraca de eficiência de mercado é contrariada, o que pode sugerir uma precificação inadequada do instrumento no mercado. Este trabalho ganha ainda mais relevância pela recente autorização de negociação de contratos de Ibovespa futuro para o mercado americano e a divulgação por parte de profissionais da área financeira do instrumento como bom provedor de hedge para operação com ações ${ }^{(2)}$.

Segundo Ross, Westerfield e Jaffe (2002), a eficiência na forma fraca é o tipo menos exigente de eficiência que podemos esperar de um mercado financeiro, pois a informação histórica sobre preços é a espécie mais fácil de informação que se pode adquirir a respeito de uma ação. Para os autores, se fosse possível obter lucros relevantes simplesmente verificando padrões de comportamento nas séries de preços de ações, todos o fariam, e qualquer lucro logo desapareceria na luta para obtê-los. Elton e Gruber (1995) afirmam que, se os testes empíricos provam ser impossível prever retornos futuros com base em retornos passados, então as táticas de negociação baseadas em exames da sequência de preços passados são inúteis.

No mercado internacional, Brock, Lakonishok e Le Baron (1992) testaram as metodologias de médias móveis e rompimento de suporte e resistência, com dados do Dow Jones Index de 1897 a 1986, e encontraram resultados favoráveis à análise técnica. Ratner e Leal (1999) examinaram os retornos obtidos com a utilização de médias móveis em 10 índices de ações de mercados emergentes na América Latina e Ásia. Os autores verificaram que três dos 10 índices analisados favoreceram a hipótese de que a análise técnica apresenta chances de sucesso. Osler e Chang (1995) avaliaram rigorosamente o poder de previsão do padrão gráfico ombro-cabeça-ombro. O trabalho foi um dos pioneiros a testar padrões gráficos, sendo seguido recentemente no Brasil por Boainain (2007) que, assim como o trabalho original, indica que o padrão ombro-cabeça-ombro consegue capturar, nas séries históricas de preços de ações, sinais a respeito da movimentação futura de preços.

No Brasil, Saffi (2003) testa a validade da hipótese dos mercados eficientes no mercado futuro do índice Ibovespa. Ao contrário de Saffi (2003), que seleciona as estratégias mais comuns de análise técnica, optamos por, além da mudança da $\operatorname{amostra}^{(3)}$, escolher uma estratégia específica de análise técnica. Enquanto Saffi (2003) procura minimizar a crítica de que a estratégia só funcionaria para a série estudada (data-snooping), mediante a técnica de bootstrap, neste trabalho, dividimos a amostra em in-sample e out-sample para ratificarmos a aplicabilidade da estratégia, após uma observação inicial. Os resultados encontrados não invalidam as conclusões do trabalho anterior, mas mostram que o derivativo em questão possibilita a exploração de operações mais sofisticadas do que as estratégias usuais e as de comprar e esperar. Sato (2002) emprega uma estratégia de market timing no mercado à 
vista da BOVESPA. Sato (2002) e Saffi (2003) mostram que a análise técnica não consegue, de forma consistente, retornos superiores. Cunha (2002), analisando os padrões de candlestick $^{(4)}$ no mercado à vista de câmbio e mercado futuro de ações e Santos, Costa e Silveira (2003), constatam padrões de dependência nas séries analisadas, dando suporte à análise técnica. Vidotto, Migliato e Zambon (2009) mostram que o indicador de análise técnica Moving Average Convergence/Divergence (MACD) é uma ferramenta eficaz ${ }^{(5)}$.

Este artigo contribui com os poucos estudos acadêmicos do mercado brasileiro sobre a validade ou não de algumas ferramentas utilizadas pela análise técnica, ao reproduzir, de maneira objetiva, a utilização de uma estratégia operacional desenvolvida pelos autores, que tem por finalidade mostrar que é possível realizar operações lucrativas baseadas em indicadores de análise técnica no mercado de futuro. Estas operações obtêm bom desempenho, mesmo quando o retorno é controlado pelo risco.

O artigo está dividido em quatro partes. Na próxima seção, é descrita a amostra, a metodologia e as estratégias utilizadas neste trabalho. Na terceira seção são apresentados os resultados e, na última, conclui-se o trabalho.

\section{Amostra, Metodologia e Estratégias}

\section{Amostra e metodologia}

A amostra utilizada são as cotações de abertura e fechamento do minicontrato do Ibovespa Futuro em períodos de 5 minutos. Os dados foram coletados do software Profitchart RT, de janeiro de 2006 a fevereiro de 2010. Todas as operações foram realizadas com 20 pontos de diferença em relação ao preço negociado: as operações de compra foram executadas 20 pontos acima do preço e as operações de venda, 20 pontos abaixo do preço ${ }^{(6)}$. Este procedimento foi feito para ilustrar um possível spread na hora da execução das ordens. Utilizamos a janela de janeiro de 2006 a dezembro de 2007 para calibrar a estratégia, e a janela de janeiro de 2008 a fevereiro de 2010 para a sua avaliação.

A estratégia foi desenvolvida para que não se carregue nenhuma posição para o dia seguinte, ou seja, toda a posição é fechada no preço de abertura da última barra do dia, observando os 20 pontos de diferença $^{(7)}$. Assim sendo, o risco do mercado overnight, que pode fazer com que os preços abram abaixo ou acima do preço de fechamento do dia anterior, é evitado.

Além disso, a estratégia implementada não utiliza target price ${ }^{(8)}$. Isso se deve ao fato de a estratégia ser desenvolvida para seguir possíveis tendências; como não sabemos quando uma tendência vai terminar, um target price descaracterizaria a estratégia.

As operações da estratégia são baseadas em indicadores que podem ser ilustrados em gráficos. Nesses gráficos são plotadas: (a) duas Médias Móveis Exponenciais (MME), uma de 18 períodos (MME 18) e outra de 45 períodos (MME 45); (b) uma Média Móvel Aritmética (MMA) de 300 períodos; e (c) uma MME de 255 períodos do indicador momentum com o período de $25^{(9)}$. A escolha dos respectivos períodos de cada indicador utilizado na estratégia foi feita por meio do período de calibragem que vai de janeiro de 2006 a dezembro de 2007. A Figura 1 apresenta as ferramentas utilizadas nas estratégias do minicontrato de Futuro do Ibovespa deste estudo. Na parte superior da tela, temos as médias móveis exponenciais e aritmética. Na parte inferior, temos a média móvel do indicador momentum. 


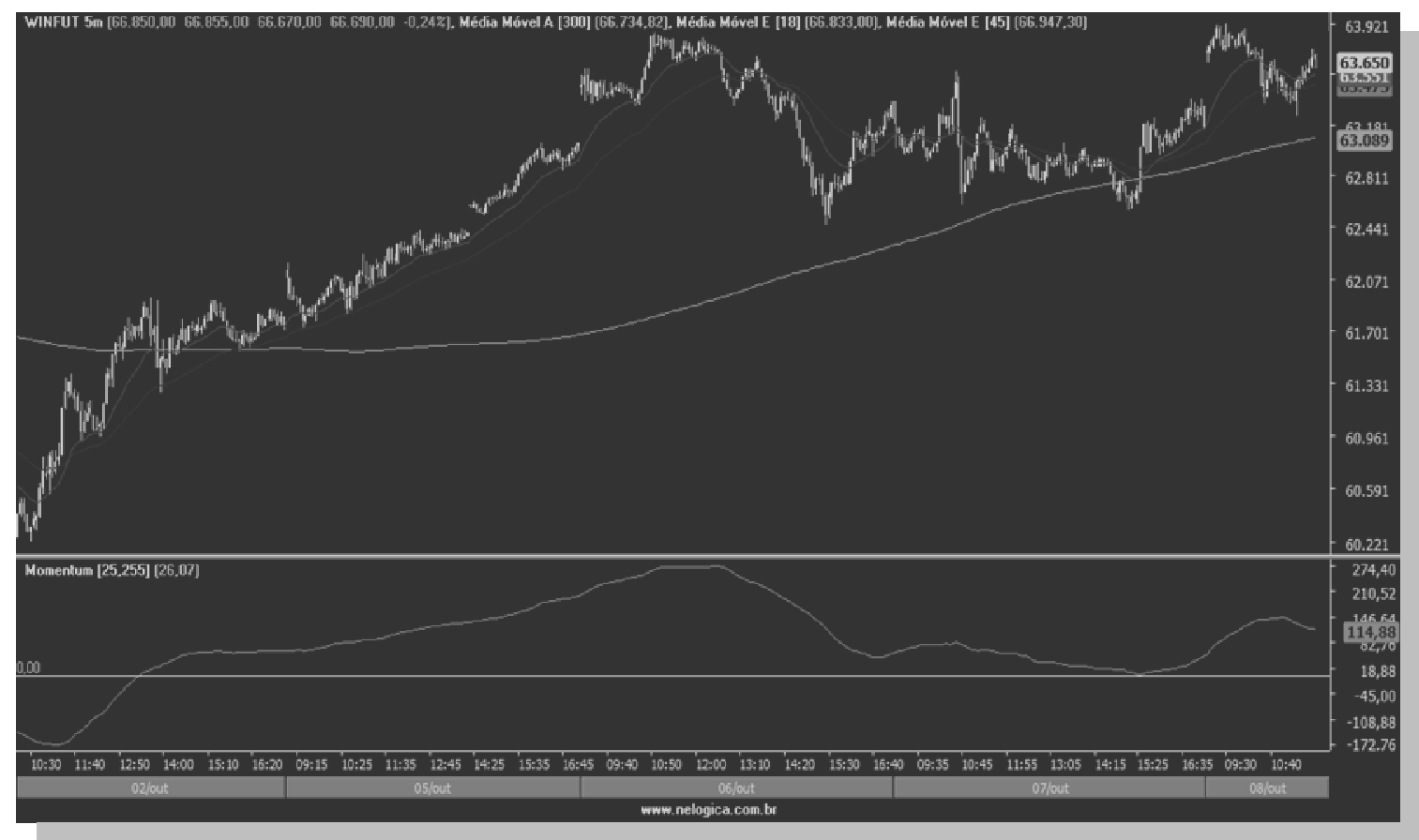

Figura 1. Ferramentas Utilizadas pela Estratégia em um Gráfico de 5 Minutos do Minicontrato de Futuro do IBOVESPA.

Na parte superior da tela (esquerda), temos a MME 18, imediatamente abaixo a MME 45 e a MMA 300 que cruza toda a tela. Na parte inferior da tela, temos a MME 255 do Momentum de 25 períodos.

\section{Estratégias de compra e venda dos contratos futuros}

Existem três cenários que devem ocorrer para a execução da compra.

Primeiro cenário. Quando a MME de 18 períodos cruzar a MME de 45 períodos de baixo para cima, a estratégia tem o sinal de compra ativado. Desta forma, o ponto de partida das estratégias, seguindo as regras de negociação de Brock et al. (1992) e Ratner e Leal (1999), é o cruzamento das médias móveis, onde o sinal de compra é indicado, quando a média móvel do período curto é maior que a do período longo.

- O sinal de compra não significa que a compra ocorre, como veremos a seguir. É importante ressaltar que a compra só acontece na abertura da barra seguinte ao cruzamento das médias, pois só com essa abertura teremos a certeza de que as médias permaneceram cruzadas ${ }^{(10)}$. A Figura 2 mostra um exemplo da ativação deste sinal de compra. 


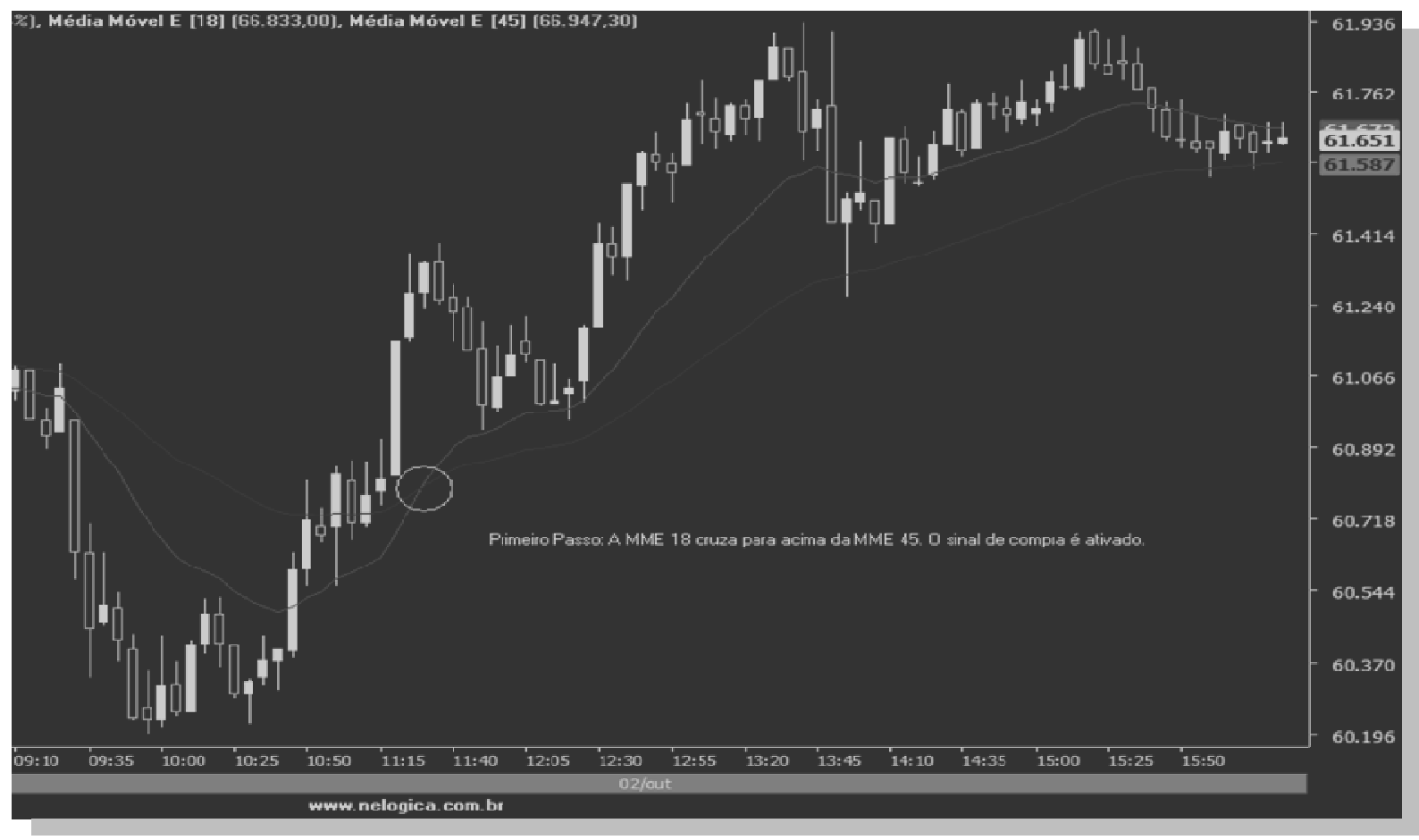

Figura 2. Ferramentas Utilizadas pela Estratégia em um Gráfico de 5 Minutos do Minicontrato de Futuro do IBOVESPA. Indicativo de Compra pelo Cruzamento da MME 18 e MME 45.

- Segundo cenário. O preço do ativo precisa estar acima da MMA de 300 períodos. Logo, caso as MMEs se cruzem (primeiro cenário), mas o preço do ativo se encontre abaixo da MMA 300, o segundo cenário só é confirmado, quando o preço de fechamento do intervalo de cinco minutos romper a MMA 300.

- Terceiro cenário. $\mathrm{O}$ indicador momentum tem que ser negativo. A intuição da estratégia é que o fato de a média móvel do período curto (ou o preço) estar maior que a do período longo sinaliza, para os analistas técnicos, que o ativo apresenta tendência altista.

A Figura 3 mostra o momento em que ocorre a compra na seta verde. Neste exemplo, o segundo passo foi o último a ocorrer.

Como a estratégia não possui um target price, a saída da operação de compra acontece em qualquer um dos dois cenários.

- Primeiro cenário. As MMEs se descruzam. Como na entrada da compra, a saída também deve respeitar a abertura da barra seguinte ao cruzamento, seja com lucro seja com prejuízo. 


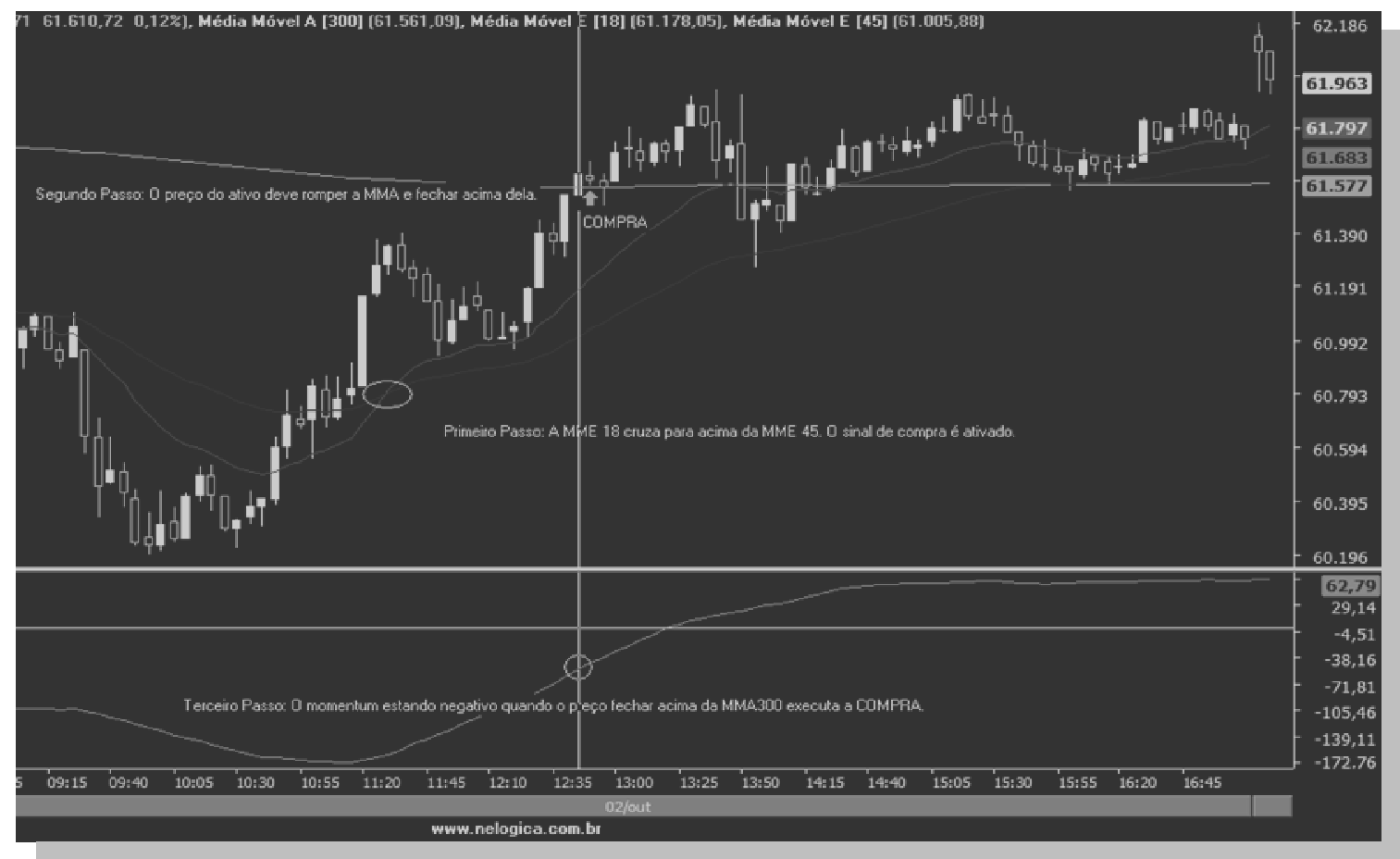

Figura 3. Ferramentas Utilizadas pela Estratégia em um Gráfico de 5 Minutos do Minicontrato de Futuro do IBOVESPA: Momento de Compra.

Na parte superior da tela (esquerda), temos a MMA 300. Logo abaixo, a MME 18 e a MME 45. Na parte inferior da tela, temos a MME 255 do Momentum de 25 períodos.

- No segundo cenário, é quando o período de negociação no dia termina. Desta forma, a saída acontece na última barra do pregão. Como já mencionado antes, a estratégia foi desenvolvida para não carregar nenhuma posição para o dia seguinte. A Figura 4, na seta vermelha, mostra um exemplo de saída na última barra do pregão.

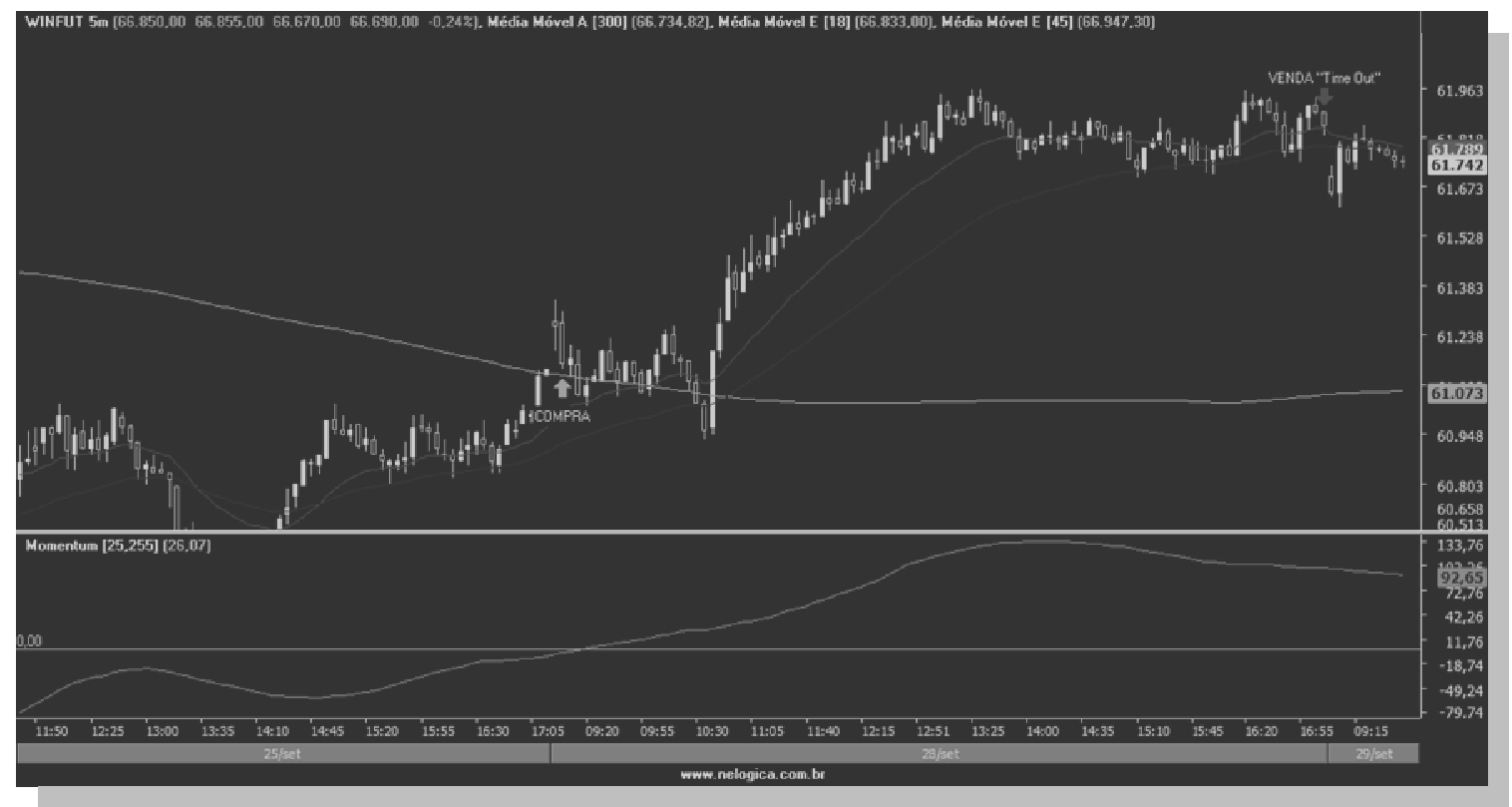

Figura 4. Ferramentas Utilizadas pela Estratégia em um Gráfico de 5 Minutos do Minicontrato de Futuro do IBOVESPA. Indicativo de Venda pela Última Barra do Pregão.

Na parte superior da tela (direita), temos a MME 18, a MME 45 e a MMA 300, respectivamente. Na parte inferior da tela, temos a MME 255 do Momentum de 25 períodos. 
A Figura 4 mostra a saída pelo término do dia, pois as MMEs não se descruzaram. É importante observar que, mesmo que o preço fique abaixo da MMA 300 e/ou o indicador de momentum esteja positivo, como demonstrado no exemplo da Figura 4, a saída só ocorre nos cenários citados.

Para a operação de venda, seguimos três cenários semelhantes aos descritos na compra.

- Primeiro cenário. Quando a MME de 18 períodos cruzar a MME de 45 períodos de cima para baixo, a estratégia tem o sinal de venda ativado.

- Segundo cenário. O preço do ativo está abaixo da MMA de 300 períodos.

- Terceiro cenário. Se os dois primeiros passos são verdadeiros, a estratégia executa a venda, caso o indicador momentum esteja positivo.

Como na compra, a saída da venda ocorre em qualquer uma das seguintes condições, explicitado a seguir.

- Primeiro cenário. As MMEs se descruzam. Como na entrada da venda, a saída também deve respeitar a abertura da barra seguinte ao cruzamento. Como na compra, dado que a estratégia não possui target price, a saída pode ser realizada com prejuízo ou lucro.

- Segundo cenário. Ocorre quando o período de negociação no dia termina.

- A Figura 5 mostra a entrada na operação de venda através da seta vermelha, e a saída da operação, no momento em que as MMEs se descruzam, através da seta verde.

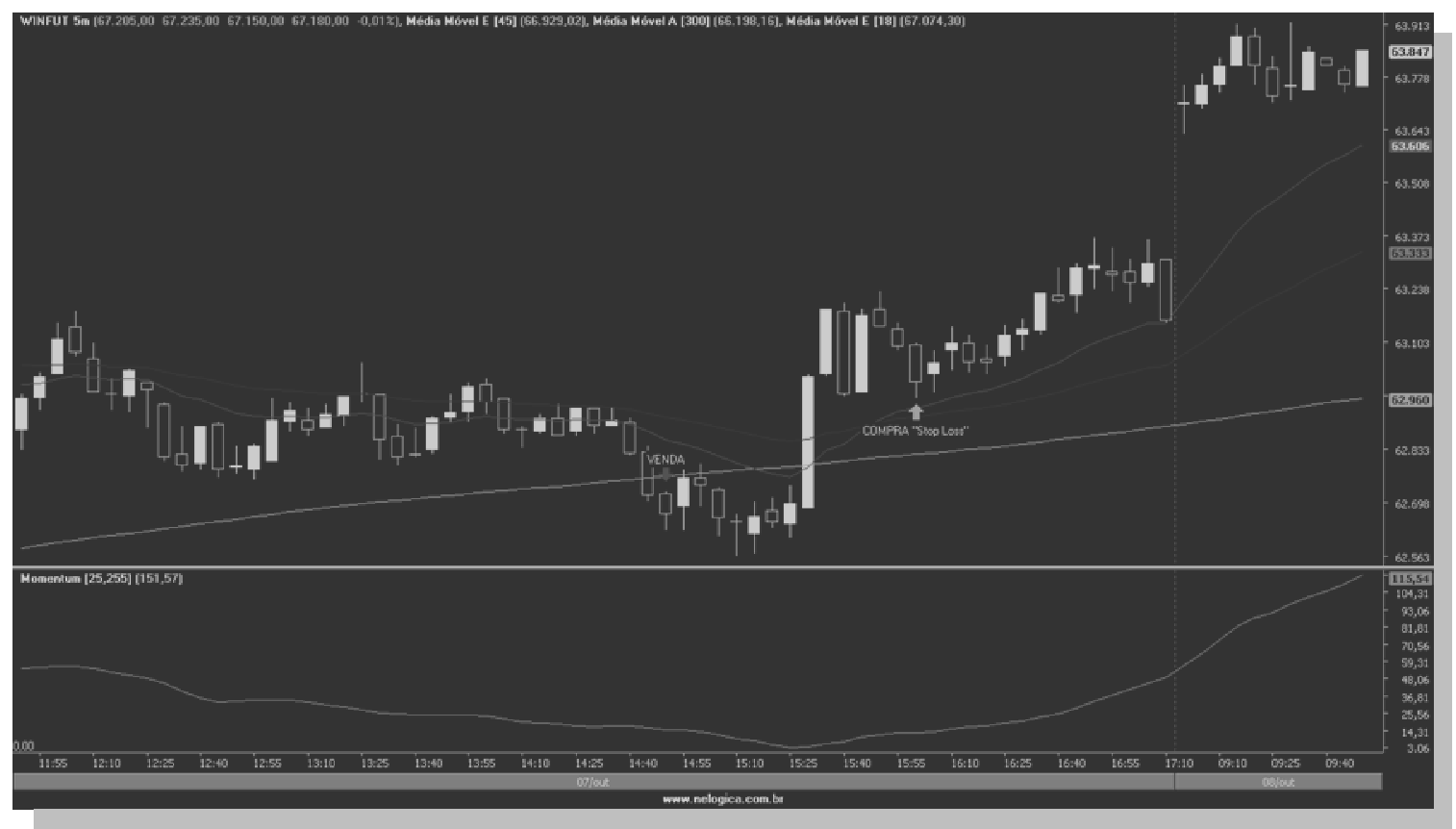

Figura 5. Ferramentas Utilizadas pela Estratégia em um Gráfico de 5 Minutos do Minicontrato de Futuro do IBOVESPA. Indicativo de Fechamento da Operação de Venda pelo Descruzamento da MME.

Na parte superior da tela (direita), temos a MME 18, a MME 45 e a MMA 300, respectivamente. Na parte inferior da tela, temos a MME 255 do Momentum de 25 períodos. 


\section{Resultados}

Nesta parte do trabalho, são analisados os resultados da estratégia no período de teste. O investimento inicial foi de $\mathrm{R} \$ 10.000,00$ (dez mil reais) e foram comprados ou vendidos três contratos por negócio, não havendo reinvestimento de capital. A escolha de três contratos por negócio foi feita conforme a margem de garantia exigida para a operação de um único contrato, que é, no período de estudo, próximo de três mil reais. Assim, em relação ao capital inicial, podemos operar o máximo de três contratos. Os $\mathrm{R} \$ 10.000,00$ (dez mil reais) gastos se referem à margem de garantia utilizada para operar os contratos ${ }^{(11)}$. O custo operacional não está incluso no cálculo da rentabilidade, pois ele varia de corretora para corretora. Na prática, participantes do mercado, que realizam estratégias semelhantes a deste trabalho, combinam uma corretagem fixa com as corretoras.

Na Tabela 1 é apresentado o resultado do sistema no período de calibragem, de janeiro de 2006 a dezembro de 2007. Na primeira coluna, temos o resultado geral da estratégia no período; na segunda coluna, é apresentado o resultado do sistema apenas para as operações de compra; na terceira coluna, temos os resultados apenas para as de venda; e a quarta coluna representa, para fins de comparação, qual seria a rentabilidade, se tivéssemos comprado e ficado (buy and hold ${ }^{(12)}$ ) com uma carteira com a mesma composição do índice Bovespa (estratégia passiva), nesse mesmo período em análise. A divisão de negócios em Somente Compra e Somente Venda mostra que a estratégia funciona em mercados de alta e de baixa.

Realizaram-se 349 operações, sendo 162 somente na compra e 187 somente na venda. O lucro obtido no período foi de $94,35 \%$ sobre o patrimônio inicial. Dentre os negócios realizados na estratégia, a média de ganhos ficou em $\mathrm{R} \$ 309,09$, enquanto a média das perdas ficou em $\mathrm{R} \$ 206,29$. Este foi o melhor resultado obtido, variando-se os parâmetros dos indicadores de análise técnica utilizados no trabalho.

Tabela 1

Resultados da Estratégia no Período de Janeiro de 2006 a Dezembro de 2007 e da Estratégia Buy and Hold no mesmo Período

\begin{tabular}{lcccc} 
& $\begin{array}{c}\text { Total } \\
\text { (Compra + Venda) }\end{array}$ & Somente Compra & Somente Venda & Buy \& Hold \\
\hline Capital Final & $\mathrm{R} \$ 19.435,00$ & $\mathrm{R} \$ 15.784,60$ & $\mathrm{R} \$ 13.650,40$ & $\mathrm{R} \$ 15.864,86$ \\
Lucro R\$ & $\mathrm{R} \$ 9.435,00$ & $\mathrm{R} \$ 5.784,60$ & $\mathrm{R} \$ 3.650,40$ & $\mathrm{R} \$ 5.864,86$ \\
Lucro ( \%) & $94,35 \%$ & $57,85 \%$ & $36,50 \%$ & $58,64 \%$ \\
Ganho anual (Capitalizado) & $39,71 \%$ & $25,82 \%$ & $16,95 \%$ & $25,95 \%$ \\
\% do Tempo Posicionado & $11,56 \%$ & $5,79 \%$ & $7,96 \%$ & $100,00 \%$ \\
Números de Negócios & 349 & 162 & 187 & 1 \\
Média de Negócios & $\mathrm{R} \$ 309,09$ & $\mathrm{R} \$ 315,01$ & $\mathrm{R} \$ 303,61$ & - \\
Vencedores & $\mathrm{R} \$-206,29$ & $\mathrm{R} \$-211,12$ & $\mathrm{R} \$-202,34$ & - \\
Média Negócios Perdedores & & & & \\
\hline
\end{tabular}

Na Tabela 2 temos o resultado no período de aplicação da estratégia, a partir da mesma estrutura da Tabela 1 . Verifica-se um lucro de $236 \%$ no período, enquanto a estratégia de comprar e segurar apresenta prejuízo. Neste período, a média diária de ganhos supera bastante a média diária de perdas. 
Tabela 2

Resultados da Estratégia no Período de Janeiro de 2008 a Fevereiro de 2010 e da Estratégia Buy and Hold no mesmo Período

\begin{tabular}{lcccc}
\cline { 2 - 5 } & $\begin{array}{c}\text { Total } \\
\text { (Compra + Venda) }\end{array}$ & Somente Compra & Somente Venda & Buy \& Hold \\
\hline Capital Final & $\mathrm{R} \$ 33.661,60$ & $\mathrm{R} \$ 23.848,60$ & $\mathrm{R} \$ 19.813,00$ & $\mathrm{R} \$ 8.772,00$ \\
Lucro R\$ & $\mathrm{R} \$ 23.661,60$ & $\mathrm{R} \$ 13.848,60$ & $\mathrm{R} \$ 9.813,00$ & $\mathrm{R} \$-1.228,00$ \\
Lucro (\%) & $236,62 \%$ & $138,49 \%$ & $98,13 \%$ & $-12,28 \%$ \\
Ganho anual (Capitalizado) & $75,82 \%$ & $49,79 \%$ & $37,42 \%$ & - \\
\% do Tempo Posicionado & $6,54 \%$ & $4,32 \%$ & $5,20 \%$ & $100,00 \%$ \\
Números de Negócios & 351 & 165 & 186 & 1 \\
Média de Negócios Vencedores & $\mathrm{R} \$ 406,47$ & $\mathrm{R} \$ 424,44$ & $\mathrm{R} \$ 388,90$ & - \\
Média de Negócios Perdedores & $\mathrm{R} \$-265,90$ & $\mathrm{R} \$-286,75$ & $\mathrm{R} \$-249,09$ & $\mathrm{R} \$-1.228,00$ \\
\hline
\end{tabular}

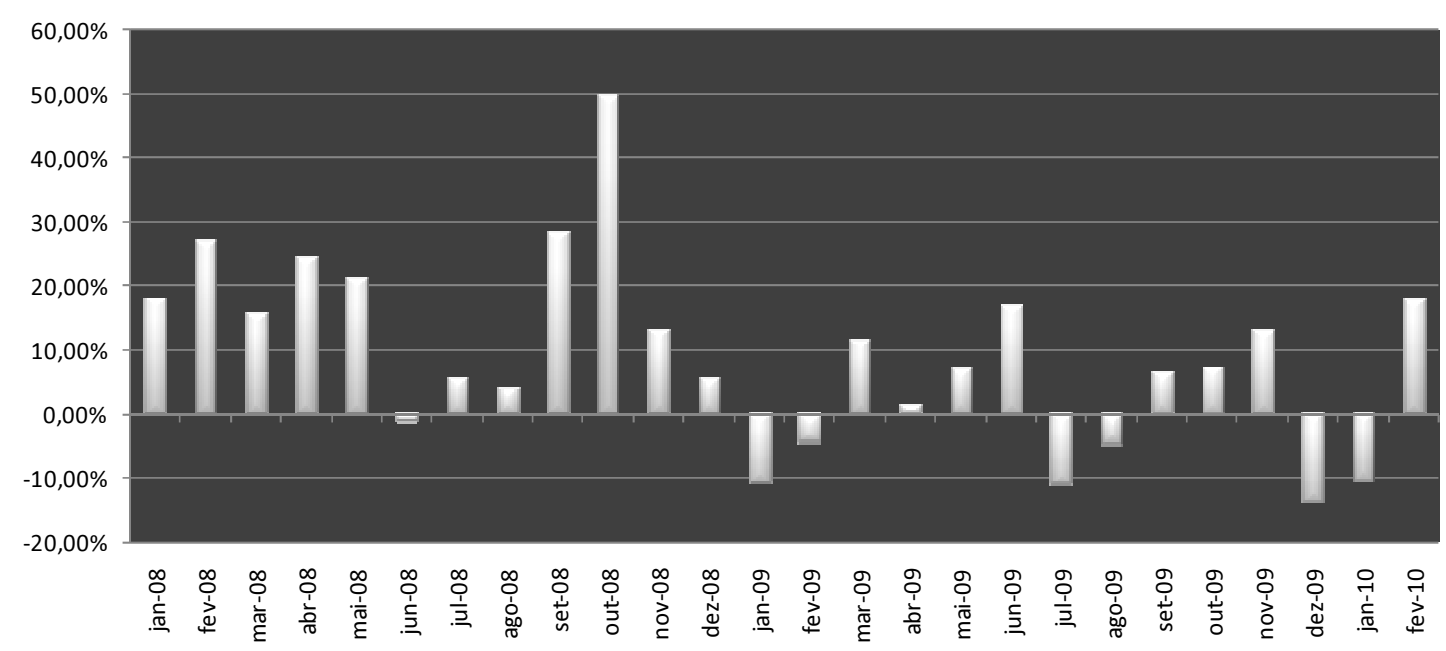

Figura 6. Gráfico de Rentabilidade Mensal.

A Figura 6 apresenta a rentabilidade mensal das estratégias. Apesar da verificação de perdas em alguns meses, como a média de ganhos financeiros é bem maior que a média de perdas financeiras, verifica-se que o resultado conjunto é positivo. Na Figura 7, temos o gráfico de dispersão dos resultados da estratégia, padronizado para um contrato por negócio. As 349 operações estão ordenadas em função do tempo no eixo das abscissas. Pode-se notar que os negócios com resultados positivos estão menos concentrados no eixo horizontal do que os negócios com resultados negativos, o que mostra que, em média, o resultado positivo é maior em termos absolutos que o resultado negativo da estratégia. 


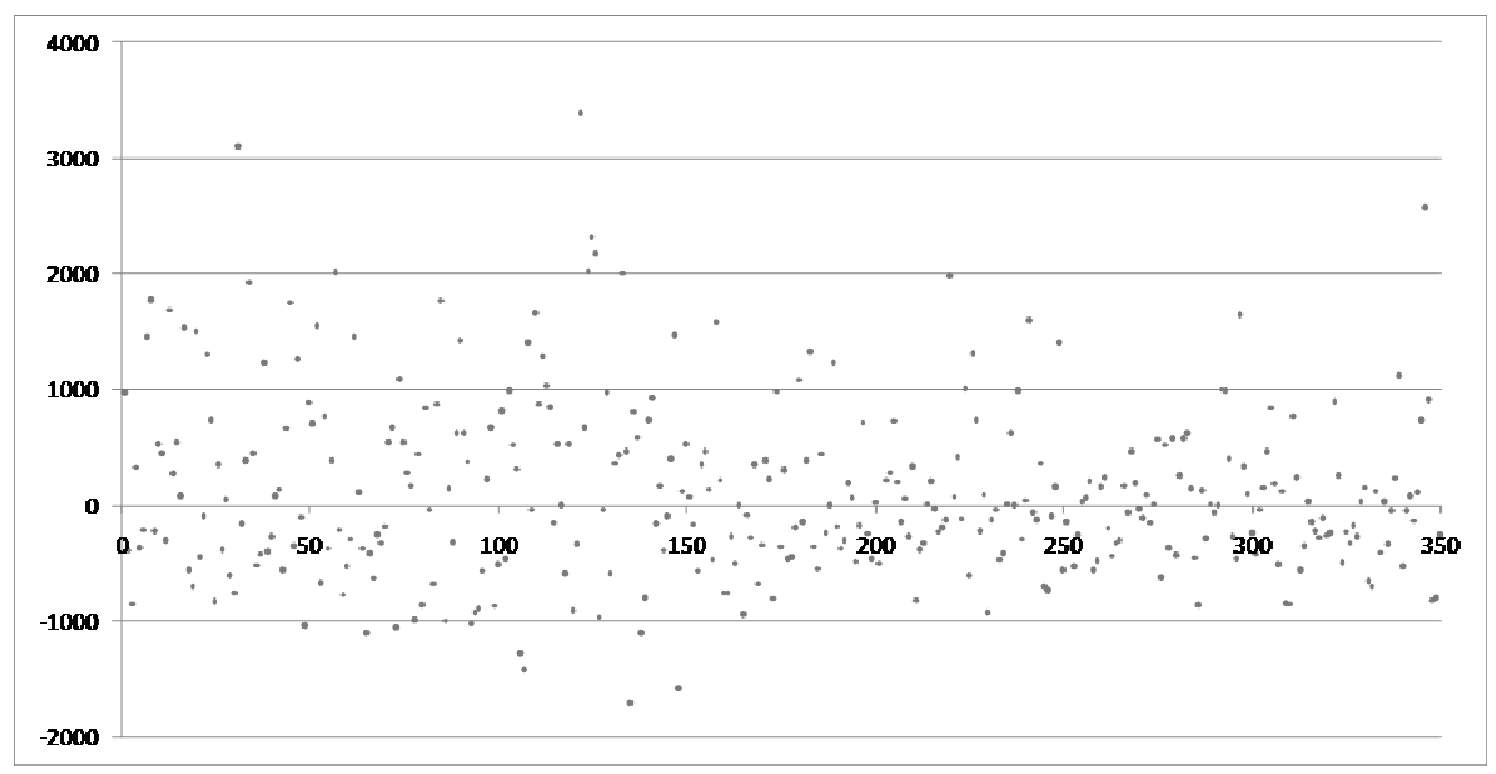

Figura 7. Gráfico de Dispersão dos Resultados por Negócio, cada Ponto Representa o Retorno em Reais, Padronizado para um Contrato, gerado por cada um dos 351 negócios.

Em relação à comparação ano a ano, percebe-se que o ano de 2008 apresentou uma relação de operações com lucro superior aos demais anos. Isso se deve ao fato de este ano ter sido marcado por fortes movimentações no mercado financeiro, representando boa oportunidade para a estratégia em determinar movimentos a serem acompanhados. A estratégia consegue seguir a tendência do mercado, utilizando as ferramentas de análise técnica e produz resultados significativos.

A fim de avaliar se a estratégia produz bons resultados, ao ajustarmos ao risco, monta-se a série de retornos diários da estratégia e do Ibovespa. Os dias em que não há negócio da estratégia são excluídos de ambas as séries. Este procedimento foi realizado para que os retornos das duas séries ficassem igualmente distribuídos ao longo do tempo. Para aferição da estratégia e do Ibovespa, calculamos seus índices de Sharpe ex post ou histórico. Neste trabalho, para a construção deste índice, seguimos Sharpe (1994): toma-se a média e o desvio-padrão do excesso de retorno diário (retorno diário da estratégia subtraído do CDI) e a razão dessas medidas é o índice de Sharpe diário ex-post. Para anualizá-lo, multiplica-se este valor por $252^{0,5}$.O índice de Sharpe da estratégia é de 2,07 contra 0,28 do Ibovespa, o que mostra a superioridade dessa estratégia de análise técnica em relação à estratégia passiva no período ${ }^{(13)}$. Para maior robustez dos resultados, reestimamos os resultados, considerando uma taxa de corretagem fixa mensal, obtida pela moda das taxas cobradas no período, no valor de $\mathrm{R} \$ 30,00$ por mês. Neste caso, o índice Sharpe anual da estratégia é de 1,97.

\section{Conclusão}

Este trabalho apresenta uma estratégia operacional, utilizando ferramentas de análise técnica e o minicontrato futuro do Ibovespa em períodos de 5 minutos, a fim de verificar se é possível auferir lucro com operações especulativas no mercado financeiro brasileiro e, assim, observar se o mercado futuro de índice apresenta a forma fraca de eficiência de mercado.

A estratégia foi desenvolvida com base nos estudos das médias móveis e do indicador momentum. A escolha dos parâmetros para cada ferramenta utilizada foi feita por uma janela de calibragem imediatamente anterior ao período estudado.

A estratégia foi realizada entre janeiro de 2008 e fevereiro de 2010, em mercados de alta e baixa, obtendo resultados interessantes, ao se analisar a sua relação retorno-risco, o que indica que o 
minicontrato futuro do Ibovespa não apresentou a forma fraca de eficiência de mercado no período estudado.

\section{Artigo recebido em 23.03.2010. Aprovado em 09.05.2011.}

\section{Notas}

${ }^{1}$ Este derivativo apresenta uma boa liquidez para o mercado brasileiro: o vencimento de janeiro apresentou, por exemplo, 46.361 negócios no dia 08/01/08, data próxima ao início da aplicação da estratégia.

${ }^{2}$ Reportagem publicada em 27/08/2009 no jornal O Globo sob o título "Estrangeiro poderá negociar Ibovespa Futuro via Globex".

${ }^{3}$ Saffi (2003) apenas utiliza preço diários (máximo, mínimo, abertura e fechamento), enquanto que, neste trabalho, os dados são intradiários.

${ }^{4} \mathrm{O}$ gráfico de candles é composto por duas partes: corpo e sombreamento. O corpo é a parte entre a abertura e o fechamento e caso a abertura tenha sido inferior ao fechamento (um dia de alta) recebe a cor branca. Se o fechamento foi menor que a abertura (um dia de queda) o corpo recebe a cor preta.

${ }^{5} \mathrm{O}$ Moving Average Convergence/Divergence é um indicador muito utilizado em análise técnica baseado em médias exponenciais.

${ }^{6}$ Por exemplo, no dia 30/10/2009, às $12 \mathrm{~h} 35$, o último negócio do minicontrato futuro de Ibovespa havia sido fechado a 63.030. Em nosso estudo, o contrato foi vendido a 63.010.

${ }^{7}$ Uma barra contém o preço de abertura, fechamento, máximo e mínimo de um período. No caso deste trabalho, o período é de 5 minutos. O preço de abertura da ultima barra do dia é o preço do primeiro negócio em um período.

${ }^{8}$ Target Price significa um preço predeterminado para sair da operação.

${ }^{9}$ A média móvel aritmética (MMA) é calculada através da média aritmética de um número fixo de preços. A cada nova informação de preço obtida, exclui-se a mais antiga. A média móvel exponencial (MME) é calculada aplicando-se um peso menor quanto menos recente é o preço do ativo. O indicador momentum é uma medida de mudança de velocidade de preço calculada como a diferença entre o preço atual e o preço de 25 períodos (de 5 minutos) atrás.

${ }^{10}$ A abertura da barra significa o preço do início da janela dos próximos 5 minutos.

${ }^{11} \mathrm{Na}$ realidade, há uma sobra dos $\mathrm{R} \$ 10.000,00$ em conta corrente para eventuais ajustes. Este capital não é investido, o que subestima os resultados da estratégia.

${ }^{12}$ A estratégia Buy and Hold representa, como seu próprio nome já diz, comprar e segurar. É um tipo de estratégia utilizada por investidores passivos ou por aqueles que desejam ter o ativo financeiro por tempo indeterminado.

${ }^{13}$ A média diária dos retornos e riscos são, respectivamente, $0,7 \%$ e 4,9\% para a estratégia contra $0,1 \%$ e 2,41\% do Ibovespa.

\section{Referências}

Boainain, P. G. (2007). Ombro-cabeça-ombro: testando a lucratividade do padrão gráfico de análise técnica no mercado de ações brasileiro (Dissertação de mestrado). IBMEC, São Paulo, SP, Brasil.

Brock, W., Lakonishok, J., \& LeBaron, B. (1992). Simple technical trading rules and the stochastic properties of stock returns. Journal of Finance, 47(5), 1731-1764. doi: 10.2307/2328994

Cowles, A. (1933). Can stock market forecasters forecast? Econometrica, 1(3), 309-324.

Cowles, A., \& Jones, H. (1937). Some a posteriori probabilities in stock market action. Econometrica, 5(3), 280-294. 
Cunha, J. (2002). Hipótese de mercados eficientes: utilização de padrões candlesticks e simulação bootstrap (Dissertação de mestrado). Universidade Federal de Santa Catarina, Florianópolis, SC, Brasil.

Elton, E. J., \& Gruber, M. J. (1995). Modern portfolio theory and investment analysis (5a ed.). New York: John Wiley \& Sons, Inc.

Fama, E. (1970). Efficient capital markets: a review of theory and empirical work. Journal of Finance, 25(2), 383-417.

Fama, E. (1991). Efficient capital markets II. Journal of Finance, 46(5), 1575-1617.

Kendall, M. (1953). The analysis of economic time series. Journal of the Royal Statistical Society, 116(1), 11-25. 11-34. doi:10.2307/2980947

Mandelbrot, B. (1966). Forecasts of future prices, unbiased markets, and "martingale" models. Journal of Business, 39(1), 242-255. doi: 10.2307/2351745

Osler, C. L., \& Chang, P. H. K. (1995). Head and shoulders: not just a flaky pattern. Federal Reserve Bank of New York, (4), 1-65.

Ratner, M., \& Leal, R. P. C. (1999). Tests of technical trading strategies in the emerging equity markets of Latin America and Asia. Journal of Banking and Finance, 23(12), 1887-1905. doi: 10.1016/S0378-4266(99)00042-4

Roberts, H. (1967). Statistical versus clinical prediction of the stock market [Working Paper]. Center for Research in Security Prices, University of Chicago, Chicago, IL, EUA.

Ross, S. A., Westerfield, R. W., \& Jaffe, J. F. (2002). Administração financeira. São Paulo: Atlas.

Saffi, P. A. C. (2003). Análise técnica: sorte ou realidade? Revista Brasileira de Economia, 57(4), 953-974. doi: 10.1590/S0034-71402003000400013

Samuelson, P. (1965). Proof that properly anticipated prices fluctuate randomly. Industrial Management Review, 6(2), 41-49.

Santos, A. A. P., Costa., N. C. A., Jr. da, \& Silveira, J. S. T. da (2003). Ganhos de eficiência e a hipótese do caminho aleatório nos mercados futuro e de ações do Brasil na década de 90. Revista Alcance, 9(1), 37-62.

Sato, A. (2002). Os riscos do market timing. Resenha BM\&F, 148, 20-24.

Sharpe, W. (1994). The sharpe ratio. Journal of Portfolio Management, 21(1), 49-58. doi: 10.3905/jpm.1994.409501.

Vidotto, R., Migliato, A., \& Zambon, A. (2009). O moving average convergence-divergence como ferramenta para a decisão de investimentos no mercado de ações. Revista de Administração Contemporânea, 13(2), 291-309. doi: 10.1590/S1415-65552009000200008

Working, H. (1934). A random difference series for use in the analysis of time series. Journal of the American Statistical Association, 29(185), 11-24. 